\title{
Two special issues
}

\section{The mini-theme of this EPN issue is complex systems. It is also the subject of two special issues of EPJ-ST.}

\section{An exploration of tipping in complex systems}

This special issue examines the extensive landscape of research into tipping within complex systems, and provides guidance as to where the field will likely be headed in the future.

Complex systems can be found in a diverse array of real-world scenarios, but are unified by their ability to suddenly transition between drastically different patterns of behaviour. Known as 'tipping, this type of transformation is generally triggered by small changes in the parameters of individual systems - whose effects can rapidly cascade to alter entire networks of interacting subsystems. In this special issue, EPJST explores the nature of tipping in complex systems through 21 new articles. Together, the studies reveal recent trends and directions of research within the field, and highlight the pressing challenges it will face in the future.

\section{Reference}

G. Ambika and J. Kurths, Tipping in complex systems: theory, methods and applications. Eur. Phys. J. Spec. Top. 230, 3177-3179 (2021). https://doi. org/10.1140/epjs/s11734-021-00281-z

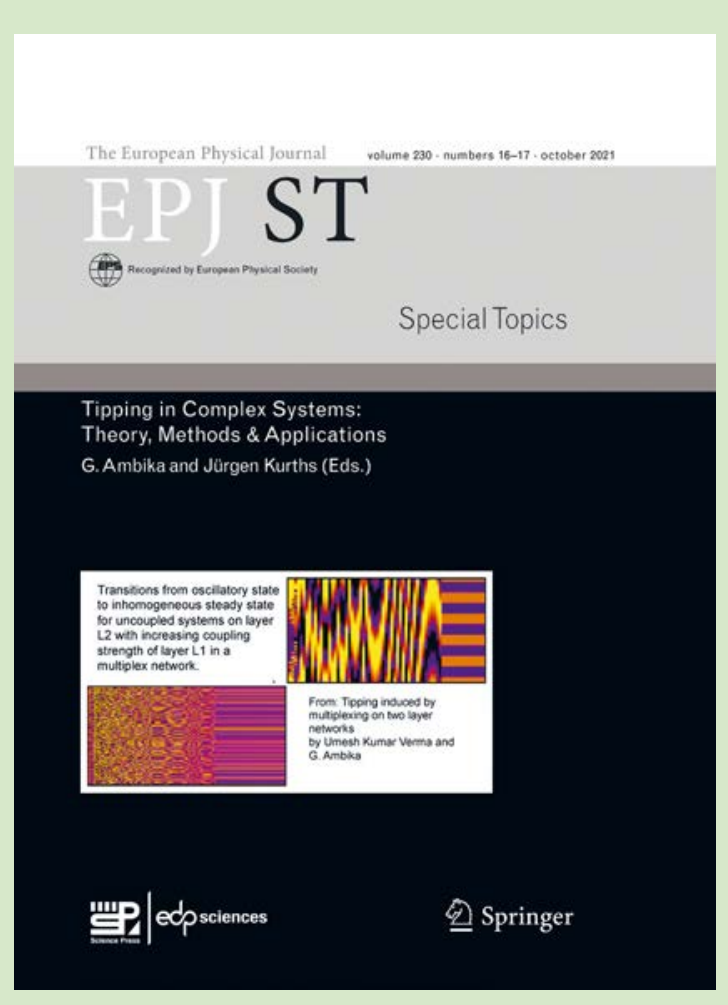

\section{Examining the dynamics of complex networks}

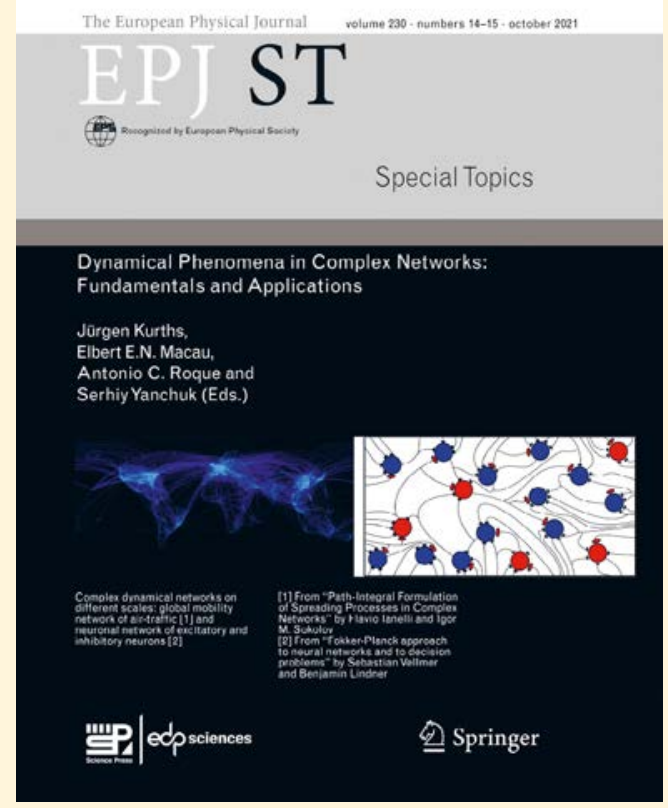

A new collection of papers focuses on the theories and methodology of dynamical networks with a focus on neuroscience and Earth sciences, and climate systems.

A special issue of EPJST, edited by Dr Jurgen Kurths, Senior Advisor at the Research Department for Complexity Science, Potsdam Institute for Climate Impact Research (PIK), and Professor and Senior Advisor at Humboldt University, Berlin, Ahmedabad, brings together a collection of papers focusing improving our understanding of the collective dynamics of complex systems. The special issue pays particular attention to the applications of this understanding in the diverse fields of neuroscience, climate modelling, and Earth science.

\section{References}

S. Yanchuk, A.C. Roque, E.E.N. Macau and J. Kurths “Dynamical phenomena in complex networks: fundamentals and applications". Eur. Phys. J. Spec. Top 